\title{
NUCLEAR POINTS IN THE THEORY OF ABSTRACT SETS*
}

\author{
BY W. SIERPINSKI
}

1. Introduction. E. W. Chittenden has published an interesting note $\dagger$ in which he gave necessary and sufficient conditions that a set $E$ contained in a class ( $V)$ (of Fréchet) be perfectly compact, or perfectly self-compact. $\ddagger$

Chittenden proves the following theorems:

THEOREM I. If an infinite set $E$ is perfectly compact, $E$ determines at least one nuclear point.

COROLLARY. Every set $E$ which is perfectly self-compact contains a nuclear point.

THEOREM II. If every infinite subset of a set $E$ of points of the space $P$ determines a nuclear point then $E$ is perfectly compact.

THEOREM III. A necessary and sufficient condition that a set $E$ be perfectly compact is that every infinite subset of $E$ determine at least one nuclear point.

COROLlary. A necessary and sufficient condition that every compact set $E$ be perfectly compact is that every infinite compact set $E$ possess at least one nuclear point.

The proofs of Chittenden are based on the following assumption ( $(3)$ : "Let $Q$ be an aggregate of power $\mu$ and of elements $q$. Of the transfinite ordinals $\Omega$ of which the aggregate of all ordinals $\alpha<\Omega$ has the power $\mu$ there is a least, $\Omega_{0}$. Let

* Presented to the Society, September 9, 1926.

$\dagger$ This Bulletin, vol. 30 (1924), p. 511.

$\ddagger$ For the definitions of the terms class $(V)$, monotone sequence, compact, perfectly compact, perfectly self-compact, limit point, nuclear point, see E. W. Chittenden, loc. cit. $\$ \S 2,4$. A topological space in which every infinite set determines a nuclear point is called by $\mathrm{P}$. Alexandroff and $\mathrm{P}$. Urysohn a bicompact space (see Mathematische ANNALEN, vol. 92 (1924), p. 260). 
(1) $q_{1}, q_{2}, q_{3}, \cdots, q_{n}, \cdots, q_{\omega}, \cdots, q_{\alpha}, \cdots, \quad\left(\alpha<\Omega_{0}\right)$

represent a 1-1 correspondence between the aggregate $Q$ and the aggregate of all ordinal numbers $\alpha<\Omega_{0}$. If $a$ sequence of ordinals $\beta<\Omega_{0}$ is determined so that for every $\alpha$ there is $a \beta>\alpha$, then the $\beta$ 's form a series of the ordinal type $\Omega_{0}$ and the aggregate of all such ordinals $\beta$ is of power $\mu . "$

This assumption is equivalent to the assumption that $\mu$ is a regular aleph. Since not every aleph is regular, the theorems of Chittenden are proved only for the sets $E$ whose power is a regular aleph.

In this note I shall examine the question: Are the theorems of Chittenden true for every set $E$ without any restriction?

2. Theorem I maybe False. Theorem I may be false for a set $E$ whose power is an irregular aleph, e. g., for

$$
\kappa_{\omega}=\sum_{n=0}^{\infty} \kappa_{n} .
$$

Let $E$ be the set of all ordinals $\xi<\omega_{\omega}$ ( $\omega_{\omega}$ denoting the least ordinal of power $\boldsymbol{\aleph}_{\omega}$ ). Let $H$ be the set of all the sequences $\xi_{1}, \xi_{2}, \xi_{3}, \cdots$ made up of different elements of $E$. Let $P=E+H$; the neighborhoods are defined as follows.

Let $\xi$ be any element of $E$ and let $\lambda<\xi$; the set of all ordinals $\eta$ such that $\lambda<\eta \leqq \xi$ is called a neighborhood of $\xi$. Let $\alpha=\left(\xi_{1}, \xi_{2}, \xi_{3}, \cdots\right)$ be any element of $H$; we call neighborhood of $\alpha$ any set composed of $\alpha$ and $\xi_{n}, \xi_{n+1}, \xi_{n+2}, \cdots$ $(n=1,2,3, \cdots)$.

Evidently every neighborhood of an element belonging to $E$ contains less than $\aleph_{\omega}$ elements of $P$, and every neighborhood of an element belonging to $H$ contains $\boldsymbol{\aleph}_{0}$ elements of $P$. Hence every neighborhood contains less than $\boldsymbol{N}_{\omega}$ elements. Thus, there does not exist any nuclear point of the set $E$ (as the power of $E$ is $\aleph_{\omega}$ ).

I shall prove that $E$ is perfectly compact. Let $S=\{G\}$ be any sequence of decreasing subsets of $E$. Let $S_{0}$ be a well-ordered sequence "confinal with $S$ " (i. e. $S_{0}$ is con- 
tained in $S$ and for each set $G$ belonging to $S$ there exists a set of $S_{0}$ contained in $\left.G\right){ }^{*}$ The sequence $S_{0}$ may be supposed of the least possible ordinal number. Moreover, we may suppose that $S$ does not contain a last element. Hence the ordinal number of $S_{0}$ is an "initial" ordinal number $\omega_{n}$, where $n$ is either 0 or a natural number. Evidently we may assume $S=S_{0}$. Consider two cases.

CASE 1. $n=0$. Hence $S=\left(G_{1}, G_{2}, G_{3}, \cdots\right)$. For each $n$, we may select an element $\alpha_{n}$ of $G_{n}$ that does not belong to $G_{n+1}$. The aggregate $\alpha=\left(\alpha_{1}, \alpha_{2}, \alpha_{3}, \cdots\right)$ is an element of $H$ and is a limit point of any sequence $\alpha_{n}, \alpha_{n+1}, \alpha_{n+2}, \cdots$. As each element of the sequence $\alpha_{n}, \alpha_{n+1}, \alpha_{n+2}, \cdots$ belongs to $G_{n}, \alpha$ is a limit point of $G_{n}(n=1,2,3, \ldots)$. Hence $\alpha$ belongs to $G^{\prime}$ for each $G$ of $S$.

CASE 2. $n \geqq 1$. In this case the set $S=\left\{G_{\xi}\right\}$ is not enumerable. Select, for each $\xi$, an element $p_{\xi}$ of $G_{\xi}$ that does not belong to $G_{\xi+1}$ (hence $p_{\xi}$ does not belong to $G_{\eta}$ if $\eta>\xi$ ). The set $Q$ of all $p_{\xi}$ is of power $\boldsymbol{\aleph}_{n}$.

Let $E_{k}$ be the set of the ordinals $\xi \leqq \omega_{k}$. Hence $E=$ $E_{1}+E_{2}+\cdots$. Hence there exists an index $k$ such that $Q$ contains $\aleph_{n}$ elements of $E_{k}$ (otherwise $Q$ would contain $\leqq \aleph_{n-1}$ elements of each $E_{k}$ and $Q$ would be of power $\leqq \boldsymbol{N}_{n-1} \cdot \boldsymbol{N}_{0}=\boldsymbol{N}_{n-1}<\boldsymbol{N}_{n}$, contrary to hypothesis). Let these elements be

$$
p_{\xi_{1}}, p_{\xi_{2}}, \cdots, p_{\xi_{\omega}}, \cdots, p_{\xi_{\alpha}}, \cdots, \quad\left(\alpha<\omega_{n}\right) .
$$

Let $\gamma$ be the least number having the property that there exists in the sequence (2) a set of power $\aleph_{n}$ of numbers $\leqq \gamma$. As the sequence (2) is contained in $E_{k}, \gamma \leqq \omega_{k}$. Now, if $\lambda<\gamma$ there is set of power $\boldsymbol{\aleph}_{n}$, composed of elements of the sequence (2) and contained between $\lambda$ and $\gamma$. It follows that, if $\alpha<\omega_{n}$, each neighborhood of $\gamma$ contains at least one element of $G_{\xi_{\alpha}}$. As the sequence $G_{\xi_{1}}, G_{\xi_{2}}, \cdots$, $G_{\xi_{\alpha}}, \cdots\left(\alpha<\omega_{n}\right)$ is confinal with $G_{1}, G_{2}, \cdots, G_{\xi}, \cdots$, it follows at once that each neighborhood of $\gamma$ contains an element of $G_{\xi}$. Hence $\gamma$ belongs to $G_{\xi}^{\prime}$ for each $\xi<\omega_{n}$.

* Hausdorff, Grundzilge der Mengenlehre, p. 132, Leipzig, Veit, 1914. 
Thus $E$ is perfectly compact and Theorem $I$ is, in this case, false. It may be proved by a similar argument that for any irregular aleph there exists a set $E$ for which the Theorem I does not hold true.

3. The Corollary of Theorem I holds True for any Set E. Since Chittenden gave a proof of the corollary in case the power of $E$, call it $\boldsymbol{\aleph}_{\alpha}$, is a regular aleph, I shall suppose that $\boldsymbol{N}_{\alpha}$ is irregular. It follows that there exists an ordinal $\beta<\alpha$ (of the second kind) and a transfinite sequence of increasing ordinals $\alpha_{\xi}<\alpha(\xi<\beta)$ such that $\lim _{\xi<\beta} \alpha_{\xi}=\alpha$ and

$$
\kappa_{\alpha}=\sum_{\xi<\beta} \aleph_{\alpha_{\xi}} \text {. }
$$

Suppose $E$ is a perfectly self-compact set that does not contain any nuclear point. Then there exists for each element $p$ of $E$ a neighborhood $V(p)$ such that the product $E \cdot V(p)$ is of power $<\aleph_{\alpha}$. Let

$$
p_{1}, p_{2}, p_{3}, \cdots, p_{\omega}, \cdots, p_{\kappa}, \cdots \quad\left(\lambda<\omega_{\alpha}\right)
$$

be the sequence (of the least possible type) constituted of all the elements of $E$.

Let $\xi<\beta$; let $T_{\xi}$ be the sum of all the sets $E \cdot V\left(p_{\lambda}\right)$ such that $E \cdot V\left(p_{\lambda}\right)$ is of power $\leqq \aleph_{\alpha_{\xi}}$ and $\lambda<\omega_{\alpha_{\xi}}$. Thus $T_{\xi}$ is obtained by adding together $\leqq \aleph_{\alpha_{\xi}}$ sets each of power $\leqq \boldsymbol{N}_{\alpha_{\xi}}$. It follows that the power of $T_{\xi}$ is $\leqq \boldsymbol{N}_{\alpha_{\xi}}^{2}=\boldsymbol{N}_{\alpha_{\xi}}<\boldsymbol{N}_{\alpha}$ (since $\xi<\beta$ implies $\alpha_{\xi}<\alpha$ ). As $E$ is of power $\aleph_{\alpha}$ there must exist in $E$ a point $q_{\xi}$ that does not belong to $T_{\xi}$. Now as the numbers $\alpha_{\xi}(\xi<\beta)$ are increasing, it follows from the definition of $T_{\xi}$ that

$$
T_{\xi} \subset T_{\eta} \text { if } \xi \leqq \eta<\beta .
$$

Let $S_{\xi}$ denote the set of points $q_{\eta}$ such that $\xi \leqq \eta<\beta$. The sets $S_{\xi}$ are decreasing. Now, $E$ is perfectly self-compact. Hence the sets $S_{\xi}$ or their derived sets $S_{\xi}^{\prime}$ have a common point $q$. As $q$ belongs to $E$ there is an ordinal $\lambda$ such that $q=p_{\lambda}$. As $\lambda<\omega_{\alpha}$ and $\lim _{\xi<\beta} \alpha_{\xi}=\alpha, \lim _{\xi<\beta} \omega_{\alpha_{\xi}}=\omega_{\alpha}$, there exists an ordinal $\rho<\beta$ such that $\lambda<\omega_{\alpha_{\rho}}$. Now, since the 
set $E \cdot V\left(p_{\lambda}\right)$ is of power $<\boldsymbol{\aleph}_{\alpha}$ there exists an ordinal $\zeta<\beta$ such that $E \cdot V\left(p_{\lambda}\right)$ is of power $\leqq \aleph_{\alpha \zeta}$. Let $\xi=\rho$ in case $\rho \geqq \zeta$ and $\xi=\zeta$ in case $\rho<\zeta$. Thus: $\xi<\beta, \lambda<\omega_{\alpha_{\xi}}$ and $E \cdot V\left(p_{\lambda}\right)$ is of power $\leqq \aleph_{\xi^{*}}$. Hence $p_{\lambda}$ belongs to $T_{\xi}$. It follows from the definition of the sequence $\left\{q_{\eta}\right\}$ and from (5) that $q_{\eta}$ does not belong to $T_{\xi}$ if $\eta \geqq \xi$. Hence $q_{\eta}$ does not belong to $V(q)$ if $\eta \geqq \xi$, since the set $E \cdot V(q)=E \cdot V\left(p_{\lambda}\right)$ is contained in $T_{\xi}$. Therefore $S_{\xi} \cdot V(q)=0$, contrary to the hypothesis that $q$ is a common point either of all the sets $S_{\xi}$ or of all the $S_{\xi}^{\prime}$.

Consequently, $E$ must contain a nuclear point.

4. Theorem II holds True for any Set E. This may be proved as follows. Let $S$ be a monotonic sequence of subsets of $E$. It may be assumed (cf. $\S 2$ ) that $S$ is a wellordered series of decreasing sets $G_{\alpha}$ and that the ordinal number of $S$ is an initial number $\omega_{\gamma}$. Let $p_{\alpha}$ be in $G_{\alpha}$ but not in $G_{\alpha+1}$. The set $H$ of all $p_{\alpha}$ is of power $\boldsymbol{\aleph}_{\gamma}$.

By hypothesis, $H$ determines a nuclear point $q$. Hence each neighborhood $V$ of $q$ contains $\boldsymbol{\aleph}_{\gamma}$ points of $H$. It follows that $V$ contains at least one point of every $G_{\alpha}$. Therefore $q$ belongs to all the $G_{\alpha}^{\prime}$. Consequently $E$ is perfectly compact.

5. Conclusions. Thus Theorem II holds true for any $E$, but Theorem I may be false if the power of $E$ is an irregular aleph. If follows at once that although the condition stated in Theorem III is sufficient, it is not necessary.

Consequently the condition stated in the Corollary of Theorem III is sufficient. However, it is not necessary.

For, let $K$ be the set of all ordinals $\xi<\omega_{\omega}$ and call neighborhood of $\xi$ any set of all ordinals $\eta$ such that $\lambda<\eta \leqq \xi$ (where $\lambda<\xi$ ). By an argument analogous to that of $\S 2$, it is easy to see that every compact subset $E$ of $K$ is perfectly compact. Evidently there does not exist in $K$ any nuclear point.

ThE UNIVERSITY OF WARSAW 